TITLE:

\title{
Semiquantal analysis of adiabatic hydrogen transfer rate
}

\author{
$\operatorname{AUTHOR}(\mathrm{S}):$
}

Sakumichi, Naoyuki; Ando, Koji

\section{CITATION:}

Sakumichi, Naoyuki ... [et al]. Semiquantal analysis of adiabatic

hydrogen transfer rate. The Journal of Chemical Physics 2008, 128(16): 164516.

\section{ISSUE DATE:}

2008-04-25

URL:

http://hdl.handle.net/2433/217058

\section{RIGHT:}

(C) 2008 AlP Publishing. This article may be downloaded for personal use only. Any other use requires prior permission of the author and AIP Publishing. The following article appeared in 'J. Chem. Phys. 128, 164516 (2008)' and may be found at http://scitation.aip.org/content/aip/journal/jcp/128/16/10.1063/1.2903746. 


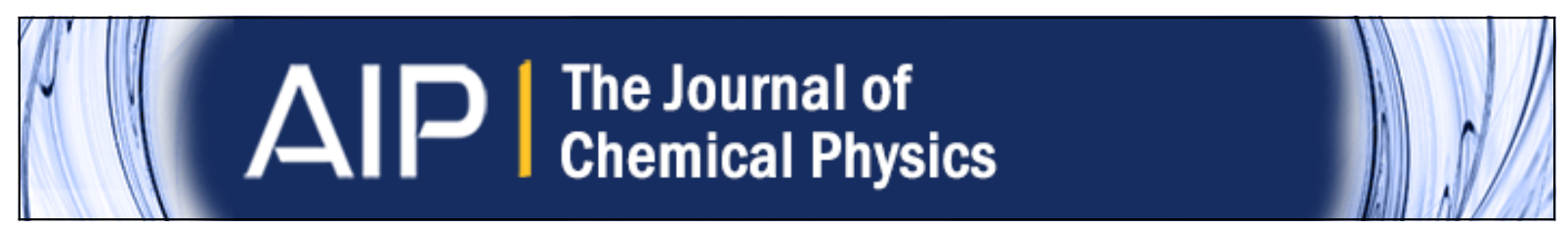

\section{Semiquantal analysis of adiabatic hydrogen transfer rate}

Naoyuki Sakumichi and Koji Ando

Citation: The Journal of Chemical Physics 128, 164516 (2008); doi: 10.1063/1.2903746

View online: http://dx.doi.org/10.1063/1.2903746

View Table of Contents: http://scitation.aip.org/content/aip/journal/jcp/128/16?ver=pdfcov

Published by the AIP Publishing

\section{Articles you may be interested in}

Barrierless proton transfer across weak $\mathrm{CH} \cdots \mathrm{O}$ hydrogen bonds in dimethyl ether dimer

J. Chem. Phys. 142, 114303 (2015); 10.1063/1.4914456

Quantum-classical Liouville dynamics of proton and deuteron transfer rates in a solvated hydrogen-bonded complex

J. Chem. Phys. 128, 164520 (2008); 10.1063/1.2907847

Efficient estimators for quantum instanton evaluation of the kinetic isotope effects: Application to the intramolecular hydrogen transfer in pentadiene

J. Chem. Phys. 127, 114309 (2007); 10.1063/1.2768930

Semiquantal analysis of hydrogen bond

J. Chem. Phys. 125, 014104 (2006); 10.1063/1.2210477

Kinetic isotope effect in hydrogen transfer arising from the effects of rotational excitation and occurrence of hydrogen tunneling in molecular systems

J. Chem. Phys. 121, 1171 (2004); 10.1063/1.1774162

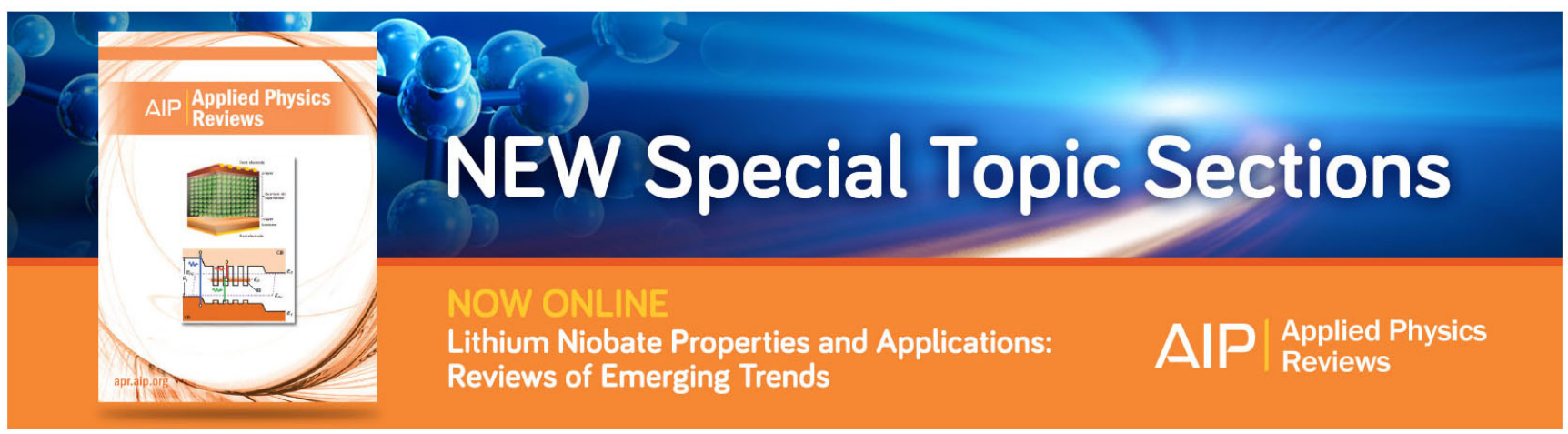




\title{
Semiquantal analysis of adiabatic hydrogen transfer rate
}

\author{
Naoyuki Sakumichia) and Koji Ando \\ Department of Chemistry, Graduate School of Science, Kyoto University, Sakyo-ku, Kyoto 606-8502, Japan
}

(Received 26 November 2007; accepted 6 March 2008; published online 25 April 2008)

\begin{abstract}
The reaction rate of adiabatic proton/hydrogen/hydride $(\mathrm{H})$ transfers in condensed phase is examined by combining the semiquantal time-dependent Hartree theory and the multidimensional transition state theory, which takes into account the zero-point effect and the dynamical modulation of the wavepacket width in the adiabatic transfer regime. By applying the theory to a model potential consisting of a quartic double well coupled linearly and quadratically (symmetrically) to external degrees of freedom, a set of compact analytical formulas was derived for the adiabatic $\mathrm{H}$ transfer rate. The analysis suggests that the kinetic isotope effect on the $\mathrm{H}$ transfer rate may exhibit a maximum as a function of the coupling strength to the external degrees of freedom measured by the reorganization energy. (C) 2008 American Institute of Physics. [DOI: 10.1063/1.2903746]
\end{abstract}

\section{INTRODUCTION}

Quantum effects are essential in determining the rate of hydrogen/proton/hydride $(\mathrm{H})$ transfer reactions in condensed phase. The mechanism may be classified into the so-called adiabatic $^{1-10}$ and nonadiabatic ${ }^{11-19}$ regimes. The prototypes of the latter include the intramolecular proton transfer isomerizations, as in malonaldehyde and tropolone, where the heavy atoms between which the $H$ transfers are constrained with regard to both the distance and the valence directions, so that the barrier along the $\mathrm{H}$ displacement is high enough to hold at least a few $\mathrm{H}$ vibrational levels to exhibit resonance tunneling splittings. On the other hand, in the adiabatic case, the positions of the heavy atoms are more flexible, as are typically seen in intermolecular $\mathrm{H}$ transfers, so that they may approach close enough by thermal fluctuation to reduce the barrier along the $\mathrm{H}$ displacement in such ways that the picture of deep tunneling no longer applies, but the $\mathrm{H}$ wavepacket adiabatically follows the modulation of the potential coupled to the environment. ${ }^{3}$ While the nonadiabatic regime has been appropriately described by the perturbation theories based on the tight-binding picture, ${ }^{14,20-25}$ which are similar to the electron transfer theories in treating the environmental reorganization, ${ }^{26-28}$ theoretical description of the adiabatic regime is not so well established. In particular, the dynamical fluctuation of the wavepacket width, affected by the shape of the potential and the coupling to the environment, ${ }^{29}$ should be treated promptly beyond the tightbinding picture.

The aim of this study is to develop a theory for the adiabatic $\mathrm{H}$ transfer rate. To this end, we combine the semiquantal time-dependent Hartree (SQTDH) theory ${ }^{29,30}$ and the multidimensional transition state theory (MTST). ${ }^{31,32}$ The latter reproduces the Grote-Hynes theory ${ }^{33,34}$ for the nonMarkovian friction effect but cannot be applied to quantum $\mathrm{H}$ transfers based on the classical mechanics. However, by

\footnotetext{
${ }^{a}$ Present address: Department of Physics, Graduate School of Science, Kyoto University, Sakyo-ku, Kyoto 606-8502, Japan. Electronic mail: sakumichi@scphys.kyoto-u.ac.jp.
}

combining with the former, which provides an effective potential including the zero-point and the wavepacket spreading effects in an extended phase space, a rate formula taking into account these quantum effects will be obtained.

The derived rate formula is then applied to examine the kinetic isotope effect (KIE) on the $\mathrm{H}$ transfer rate. The KIE has been mostly studied for the nonadiabatic H transfers ${ }^{35-39}$ rather than for the adiabatic cases presumably because the effect is normally more prominent in the former. Nonetheless, our analysis suggests an intriguing qualitative behavior in the adiabatic KIE: It may exhibit a maximum as a function of the coupling strength to the environmental degrees of freedom, which is expressed in terms of the zero-time friction in the generalized Langevin equation formalism and is measured by the reorganization energy.

In Sec. II, the SQTDH theory is summarized. We present in Sec. III a set of rate formulas and examine its natures both qualitatively and via numerical calculations. The KIE on the adiabatic $\mathrm{H}$ transfer rate is studied in Sec. IV. Section V concludes with a summary and perspectives.

\section{SQTDH THEORY}

The SQTDH theory has been described previously. ${ }^{29,30}$ For the wavefunction, it assumes a Hartree product of the squeezed coherent state Gaussian wavepackets characterized by the center and width parameters. The equations of motion for the parameters are determined from the time-dependent variational principle, ${ }^{40,41}$ which yields the equations of motion for both the center and width parameters in the form of the classical Hamiltonian equations of motion. ${ }^{42-44}$ We may thus extend the phase space to include the width coordinates and their conjugate momenta in addition to the center coordinates. The wavepacket dynamics is viewed as a classical motion on an effective potential in the extended phase space. The theory has been examined on a quartic double-well potential $^{29,30}$ and the coupled Morse and the LippincottSchroeder models of hydrogen bond. ${ }^{45,46}$

For simplicity, we set $\hbar=1$ and assume that the coordi- 
nates $q_{1}, \ldots, q_{f}$ have been mass weighted. The trial wavefunction under the time-dependent Hartree ansatz is given by

$$
\begin{aligned}
\Psi\left(q_{1}, \ldots, q_{f}, t\right)= & \prod_{i=1}^{f} N_{i} \exp \left[A_{i}(t)\left(q_{i}-x_{i}(t)\right)^{2}\right. \\
& \left.+i p_{i}(t)\left(q_{i}-x_{i}(t)\right)\right]
\end{aligned}
$$

in which $N_{i}$ is the normalization factor and

$$
A_{i}(t)=\frac{-1+2 i \delta_{i}(t) \pi_{i}(t)}{4 \delta_{i}(t)^{2}}
$$

The time-dependent parameters $x_{i}(t)$ and $\delta_{i}(t)$ describe the centers and widths of the wavepackets. The parameters $p_{i}(t)$ and $\pi_{i}(t)$ correspond to the conjugate momenta of $x_{i}(t)$ and $\delta_{i}(t)$, respectively. ${ }^{29}$ Subject to the ansatz, the situations in which the wavepacket description is inadequate, in particular, the nonadiabatic resonance tunneling case in a deep double well, are beyond the scope of this approximation. The problem of the TDH approach to the nonadiabatic $\mathrm{H}$ transfers coupled to environmental degrees of freedom has been previously examined. ${ }^{47}$ The critical point is whether the bath responds to the average force from the system ( $\mathrm{H}$ transfer) coordinate or a force different for different values of the system coordinate. This suggests that the extension to the multiconfiguration ansatz is needed for the nonadiabatic regime but would be mitigated for the adiabatic cases where the $\mathrm{H}$ wavepacket is delocalized over the low barrier along the transfer coordinate, e.g., in strong hydrogen bonds. ${ }^{4,46}$

The time-dependent variational theory applied to the trial wavefunction yields the equations of motion having the classical Hamiltonian form,

$$
\begin{aligned}
& \dot{x}_{i}=\partial H_{\mathrm{ext}} / \partial p_{i}, \quad \dot{p}_{i}=-\partial H_{\mathrm{ext}} / \partial x_{i}, \\
& \dot{\delta}_{i}=\partial H_{\mathrm{ext}} / \partial \pi_{i}, \quad \dot{\pi}_{i}=-\partial H_{\mathrm{ext}} / \partial \delta_{i},
\end{aligned}
$$

with the extended Hamiltonian defined by

$$
H_{\mathrm{ext}}\left(x_{i}, \delta_{i}, p_{i}, \pi_{i}\right)=\sum_{i=1}^{f}\left(\frac{p_{i}^{2}}{2}+\frac{\pi_{i}^{2}}{2}+\frac{1}{8 \delta_{i}^{2}}\right)+\langle V\rangle\left(x_{i}, \delta_{i}\right),
$$

in which $\langle V\rangle$ is the expectation value of the potential,

$$
\begin{aligned}
\langle V\rangle\left(x_{i}, \delta_{i}\right)= & \left(2 \pi \delta^{2}\right)^{-1 / 2} \int \prod_{i=1}^{f} d q_{i} \\
& \times \exp \left(-\frac{1}{2} \frac{\left(q_{i}-x_{i}\right)^{2}}{\delta_{i}^{2}}\right) V\left(q_{1}, \ldots, q_{f}\right) .
\end{aligned}
$$

This $\langle V\rangle$ includes nonlocal contributions due to the spreading of the wavepacket, which will be seen in the analysis in the next section to be the origin of the barrier lowering at the saddle point. The quantum dynamics is thus investigated in an extended phase space formed by $\left(x_{i}, \delta_{i}, p_{i}, \pi_{i}\right)$. The key quantity is the extended potential,

$$
V_{\mathrm{ext}}\left(x_{i}, \delta_{i}\right)=\sum_{i=1}^{f} \frac{\hbar^{2}}{8 m_{i} \delta_{i}^{2}}+\langle V\rangle\left(x_{i}, \delta_{i}\right),
$$

which is defined in the configuration space $\left(x_{i}, \delta_{i}\right)$. Note that $\hbar$ and the atomic masses $m_{i}$ have been retrieved in the first term, which tends to broaden the wavepacket by forming a potential wall developing along $\delta \rightarrow+0$, proportional to the inverse of the mass, and vanishes in the classical limit $\hbar \rightarrow 0$. The combination of this term and $\langle V\rangle$ yields the zeropoint energies. $^{29}$

It would be interesting to note that the fourth-order Taylor expansion of $\langle V\rangle$ gives an equivalent of the quantized Hamilton dynamics-2 (QHD-2) theory ${ }^{48,49}$ derived from mixed quantum-classical Heisenberg equations of motion. It has been shown that the QHD-2 theory can reproduce quantum tunneling from the cubic metastable potential reasonably well, which suggests that our SQTDH can also capture tunneling contributions near the barrier top. We also note that the QHD is recently extended to the quantized cumulant dynamics theory ${ }^{50}$ by taking into account the higher order contributions via the cumulant expansion technique.

\section{SEMIQUANTAL GROTE-HYNES THEORY}

\section{A. Model potential}

For a model of $\mathrm{H}$ transfers in condensed phase, we consider a double-well potential $V_{0}(q)$ with a symmetrically coupled mode $Q$ and a collection of linearly coupled bath modes $Q_{i}$,

$$
\begin{aligned}
V\left(q, Q, Q_{i}\right)= & V_{0}(q)+\frac{M \Omega^{2}}{2}\left(Q-\frac{D}{M \Omega^{2}} q^{2}\right)^{2} \\
& +\sum_{i} \frac{M_{i} \Omega_{i}^{2}}{2}\left(Q_{i}-\frac{C_{i}}{M_{i} \Omega_{i}^{2}} q\right)^{2},
\end{aligned}
$$

$$
V_{0}(q)=-\frac{a}{2} q^{2}+\frac{b}{4} q^{4}
$$

This extends the well-studied "double-well plus harmonic bath" model $^{32,51-53}$ by adding the mode $Q$ representing the displacements of the heavy atoms between which the $\mathrm{H}$ transfers. Its importance has been noted in previous works, ${ }^{21,54-59}$ mostly in the context of the nonadiabatic $\mathrm{H}$ transfers. The linearly coupled modes $Q_{i}$ represent the environmental polarization, whose equilibrium configurations depend on the $\mathrm{H}$ transfer coordinate $q$. By collectively describing the $Q_{i}$ in terms of the diabatic energy gap coordinate, it has been shown by combined $a b$ initio molecular orbital and Monte Carlo calculations that the model of Eq. (7) adequately describes the solvent couplings to the acid $(\mathrm{HCl}$ and $\mathrm{HF}$ ) ionization proton transfers in water. ${ }^{60,61}$

By applying the SQTDH theory to Eq. (7), the extended potential is derived as 


$$
\begin{aligned}
V_{\mathrm{ext}}\left(x, X, X_{i} ; \delta, \Delta, \Delta_{i}\right)= & \langle V\rangle\left(x, X, X_{i} ; \delta, \Delta, \Delta_{i}\right)+\frac{\hbar^{2}}{8 m \delta^{2}} \\
& +\frac{\hbar^{2}}{8 M \Delta^{2}}+\sum_{i} \frac{\hbar^{2}}{8 M_{i} \Delta_{i}^{2}},
\end{aligned}
$$

in which $\left(x, X, X_{i}\right)$ and $\left(\delta, \Delta, \Delta_{i}\right)$ are the wavepacket centers and widths for $\left(q, Q, Q_{i}\right)$, and

$$
\begin{aligned}
\langle V\rangle\left(x, X, X_{i} ; \delta, \Delta, \Delta_{i}\right) \\
=V\left(x, X, X_{i}\right)+\frac{1}{2} M \Omega^{2} \Delta^{2}+\sum_{i} \frac{1}{2} M_{i} \Omega_{i}^{2} \Delta_{i}^{2} \\
+\left(-D X+\frac{3}{2} b x^{2}+\frac{3 D^{2}}{M \Omega^{2}} x^{2}-\frac{a}{2}+\frac{1}{2} m \zeta(0)\right) \delta^{2} \\
+\frac{3}{4}\left(b+\frac{2^{2}}{M \Omega^{2}}\right) \delta^{4} .
\end{aligned}
$$

The linearly coupled modes are represented by the parameter $\zeta(0)$ defined by $m \zeta(0) \equiv \Sigma_{i} C_{i}^{2} / M_{i} \Omega_{i}^{2}$, which stems from the time-dependent friction kernel $m \zeta(t) \equiv \Sigma_{i}\left(C_{i}^{2} / M_{i} \Omega_{i}^{2}\right) \cos \Omega_{i} t$ in the semiquantal generalized Langevin equations. ${ }^{29,30}$ It is also related to the reorganization energy, as shown in Sec. III C.

\section{B. Analytical rate formulas}

The analysis of the reactant well bottom and the saddle point of $V_{\text {ext }}$ in the framework of the MTST yields the reaction rate taking into account the quantum effects via the SQTDH model. We assume that the Hessian matrices should be derived from the function $V_{\text {ext }}\left(x, X, X_{i}\right)$ obtained by minimizing along the wavepacket width coordinates $\delta, \Delta$, and $\left\{\Delta_{i}\right\}$. An analogous "local adiabatic elimination" of the fluctuating coordinates has been examined ${ }^{62}$ to find excited states via the Einstein-Bohr-Sommerfeld-type 'requantization' of the wavepacket dynamics. The details on the well bottom and the saddle point and their Hessian matrices are described in the Appendix. Compact analytical formulas are derived in weak and strong bath coupling cases given by $m \zeta(0) \ll a$ and $m \zeta(0) \gg a$, respectively.

Weak bath coupling case. The semiquantal reaction rate for the weak coupling case $[m \zeta(0) \ll a]$ is derived as

$$
k_{\mathrm{SQGH}}^{\text {weak }}=\frac{\mu_{\text {weak }}}{2 \pi} \sqrt{\frac{B^{\prime}}{B} \frac{2 a-3 B \hbar / \sqrt{m \alpha}}{m \zeta(0)}} \exp \left(-\beta E_{b, \text { weak }}^{*}\right),
$$

in which $B=b+4 D^{2} / 3 M \Omega^{2}, \quad B^{\prime}=b+2 D^{2} / M \Omega^{2}, \quad$ and $\alpha=2 a B^{\prime} / b+m \zeta(0)$. These may be viewed as the coefficients $a$ and $b$ in $V_{0}$ affected by the coupling to the external modes. The effective barrier height in Eq. (11) is given by

$$
E_{b, \text { weak }}^{*}=E_{b}-\frac{(a-m \zeta(0))^{2}}{12 B}-\frac{\hbar}{2} \sqrt{\frac{\alpha}{m}},
$$

where $E_{b} \equiv a^{2} / 4 b$ corresponds to the classical barrier height. It is reduced by the second and third terms due to the quantum effects. The second term represents the saddle point lowering, while the third term comes from the zero-point energy in the reactant well. The second term does not vanish in the limit $\hbar \rightarrow 0$, as the width of the wavepacket remains finite at the saddle point of the double-well potential (Appendix). Consequently, the effective potential at the saddle point captures the nonlocal contributions via Eq. (5) to acquire the lower energy than the classical value. This is robust in the current framework as the more accurate approximation only introduces terms of order $\hbar^{2}$ and higher. ${ }^{63}$ Interestingly, the present picture on the barrier lowering seems to be different from those based on the centroid path-integral approaches ${ }^{64,65}$ in that the former does not vanish as $\hbar \rightarrow 0$. However, the picture depends on the coupling strength, such that, on the other hand, in the strong coupling case examined below [Eq. (16)], the semiquantal barrier height reduces to the classical one as $\hbar \rightarrow 0$. $^{66}$

Basically, both the second and third terms of Eq. (12) originate from the zero-point effects at the well bottom and the saddle point, apart from the differences in details as discussed above, in particular, the essential roles of the wavepacket broadening in the former. In this regard, the basic picture should be in accord with those from the previous simulations. ${ }^{2,3,60,61}$

The reactive frequency $\mu_{\text {weak }}$ in Eq. (11) is obtained as the solution of

$$
\mu=\sqrt{\frac{\hat{\zeta}^{2}(\mu)}{4}+\zeta(0)}-\frac{\hat{\zeta}(\mu)}{2},
$$

where $\hat{\zeta}$ is the Laplace transformed friction kernel,

$$
m \hat{\zeta}(z)=\sum_{i} \frac{C_{i}^{2}}{M_{i} \Omega_{i}^{2}}\left(\frac{z}{z^{2}+\Omega_{i}^{2}}\right)
$$

It is noted that Eq. (13) is slightly different from the classical Grote-Hynes equation in that the square of the barrier frequency is replaced by $\zeta(0)$.

Strong bath coupling case. In the strong coupling case with $m \zeta(0) \gg a$, we find

$$
k_{\mathrm{SQGH}}^{\mathrm{str}}=\frac{\mu_{\mathrm{str}}}{2 \pi} \sqrt{\frac{4 a-6 B \hbar / \sqrt{m \alpha}}{2 a-3 B \hbar / \sqrt{m(m \zeta(0)-a)}}} \exp \left(-\beta E_{b, \mathrm{str}}^{*}\right),
$$

in which

$$
E_{b, \mathrm{str}}^{*}=E_{b}+\frac{\hbar}{2} \sqrt{\frac{m \zeta(0)-a}{m}}-\frac{\hbar}{2} \sqrt{\frac{\alpha}{m}} .
$$

In contrast to the weak coupling case, $E_{b \text { str }}^{*}$ reduces to $E_{b}$ in the limit $\hbar \rightarrow 0$. This pertains to the fact that the determining factor here is the bath coupling which tends to suppress the wavepacket spreading. ${ }^{29}$ The reactive frequency $\mu_{\text {str }}$ is given as the solution of

$$
\mu=\sqrt{\frac{\hat{\zeta}^{2}(\mu)}{4}+\frac{1}{m}\left(a-\frac{3 B \hbar}{2 \sqrt{m(m \zeta(0)-a)}}\right)}-\frac{\hat{\zeta}(\mu)}{2} .
$$


Again, in contrast to the weak coupling case, $\mu_{\text {str }}$ includes the quantum correction of order $\hbar^{1}$ to the classical GroteHynes theory. This correction vanishes in the limit $\hbar \rightarrow 0$, reducing to the classical Grote-Hynes result as $a / m$ corresponds to the square of the classical barrier frequency.

\section{Converting the parameters to dimension of energy}

Equations (11)-(17) present the first main result of this work. However, it would be useful to convert the rather obscure parameters such as $a, b, D$, and $\zeta(0)$ to quantities of well-characterized dimensions. We thus convert them to the dimension of energy via

$$
\begin{gathered}
\omega=\sqrt{\frac{2 a}{m}, \quad E_{\lambda}=\frac{2 a}{b} m \zeta(0),} \\
E_{b}^{*}=\frac{a^{2}}{4\left(b+2 D^{2} / M \Omega^{2}\right)}=\frac{a^{2}}{4 B^{\prime}},
\end{gathered}
$$

in which $\omega$ is the frequency at the well bottom, $E_{\lambda}$ is the reorganization energy of the linearly coupled bath modes, and $E_{b}^{\ddagger}$ is the barrier height in the section of $V_{\text {ext }}(q, Q)$ at $Q=0$ which reflects the coupling strength $D$ of the symmetrically coupled mode.

The semiquantal barrier height is thus rewritten in the weak coupling case $\left(E_{\lambda} / E_{b}<8\right)$ as

$$
E_{b, \text { weak }}^{*}=E_{b}-\frac{E_{b}\left(1-E_{\lambda} / 8 E_{b}\right)^{2}}{1+2 E_{b} / E_{b}^{\ddagger}}-\frac{\hbar \omega}{2} \sqrt{\frac{E_{b}}{E_{b}^{*}}+\frac{1}{16} \frac{E_{\lambda}}{E_{b}}} .
$$

In the strong coupling case $\left(E_{\lambda} / E_{b}>8\right)$, we find

$$
E_{b, \mathrm{str}}^{*}=E_{b}+\frac{\hbar \omega}{2}\left[\sqrt{\frac{1}{2}\left(\frac{1}{8} \frac{E_{\lambda}}{E_{b}}-1\right)}-\sqrt{\frac{E_{b}}{E_{b}^{\dagger}}+\frac{1}{16} \frac{E_{\lambda}}{E_{b}}}\right] .
$$

The prefactors in Eqs. (11) and (15) can be similarly converted.

Equations (19) and (20) show an interesting dependence on the parameters $E_{b}^{*}$ and $E_{\lambda}$. When $E_{\lambda}$ is large, Eq. (20) is approximated as

$$
E_{b, \mathrm{str}}^{*} \simeq E_{b}-\frac{\hbar \omega}{2} \sqrt{\frac{E_{b}}{E_{\lambda}}}\left(1+\frac{2 E_{b}}{E_{b}^{\dagger}}\right),
$$

indicating monotonic increase in $E_{b}^{*}$ along $E_{b}^{\ddagger}$. On the other hand, when $E_{\lambda}$ is small, Eq. (19) gives

$$
E_{b, \text { weak }}^{*} \simeq E_{b}-\frac{E_{b}}{1+2 E_{b} / E_{b}^{\ddagger}}-\frac{\hbar \omega}{2} \sqrt{\frac{E_{b}}{E_{b}^{*}}},
$$

in which the second and third terms may compete as functions of $E_{b}^{\ddagger}$. It follows that the behavior of $E_{b}^{*}$ along $E_{b}^{\ddagger}$ depends on the magnitude of $\omega$. In particular, when $\omega$ is small, the dependence of $E_{b}^{*}$ on $E_{b}^{*}$ changes from decreasing to increasing functions as $E_{\lambda}$ becomes larger. This is seen in the numerical solutions in Fig. 1 discussed in the next section.
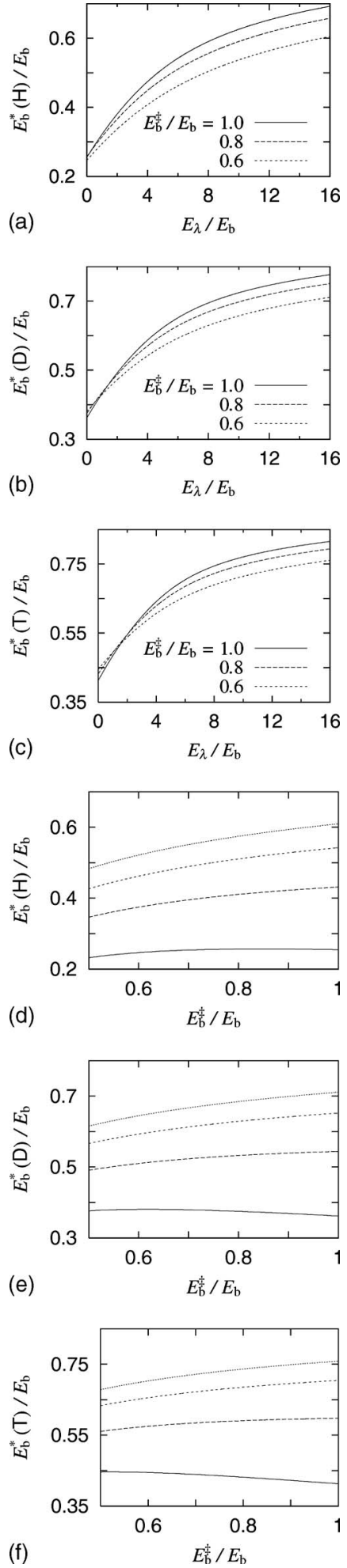

FIG. 1. Dependence of the semiquantal barrier height $E_{b \text { on }}^{*}$ on the parameters $E_{\lambda}[(\mathrm{a})-(\mathrm{c})]$ and $E_{\vec{b}}^{\ddagger}[(\mathrm{d})-(\mathrm{f})]$ for hydrogen [(a) and (d) ${ }^{b}$, deuterium [(b) and (e)], and tritium [(c) and (f)] for which $\hbar \omega / E_{b}=0.9542,0.6747$, and 0.5509 , respectively. In (d)-(f), the values of $E_{\lambda} / E_{b}$ are 0.0 (solid), 3.0 (long dashed), 6.0 (short dashed), and 9.0 (dotted).

\section{Numerical solution of the barrier height}

The compact analytical expressions presented in the previous section may be useful for qualitative discussions. Nonetheless, numerical solutions are straightforwardly obtained by finding the minima of Eqs. (A2) and (A5) in the 


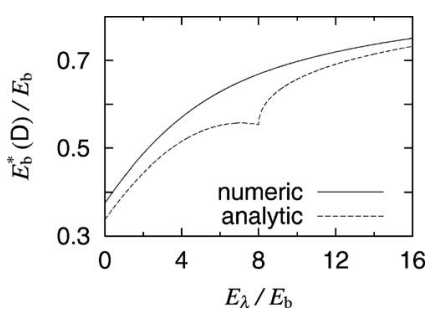

FIG. 2. Comparison between the analytical and numerical solutions for the deuterium with $E_{b}^{\ddagger} / E_{b}=0.8$

Appendix. With the energy parameters introduced in the previous section, the relevant dimensionless parameters are $E_{\lambda} / E_{b}, E_{b}^{*} / E_{b}$, and $\hbar \omega / E_{b}$, among which $\hbar \omega / E_{b}$ is directly related to the isotope effect. The other parameters such as $a$ and $E_{b}$ may be regarded to be independent of the isotopes. The numerical calculations were carried out for hydrogen $(\mathrm{H})$, deuterium (D), and tritium (T) with $\hbar \omega / E_{b}=0.9542$, 0.6747 , and 0.5509 , respectively. These numbers were chosen such that the deuterium corresponds to the intermediate barrier case in Ref. 30 and the others satisfy the ratio $\omega_{\mathrm{H}}: \omega_{\mathrm{D}}: \omega_{\mathrm{T}}=1 / \sqrt{m_{\mathrm{H}}}: 1 / \sqrt{m_{\mathrm{D}}}: 1 / \sqrt{m_{\mathrm{T}}}=1: 1 / \sqrt{2}: 1 / \sqrt{3}, \quad$ and that the adiabatic regime applies. The choice thus contains some arbitrariness rather than being designed for specific systems. We anticipate that some additional considerations, e.g., on the necessity of the "counter terms" in Eq. (7), $C_{i}^{2} q^{2} / 2 M_{i} \Omega_{i}^{2}$, would be needed for examinations of particular chemical systems.

The results of the calculations are displayed in Fig. 1. It is seen that the barrier height increases along the coupling strength to the bath. It is also noted that different behaviors among the isotopes are seen in the small coupling region of $E_{\lambda} / E_{b} \leqslant 4$. On the other hand, the barrier decreases along the parameter $D$ except for the small bath coupling limit $\left(E_{\lambda}\right.$ $=0$ ). The qualitative discussions in the previous section about the dependence of $E_{b}^{*}$ on $E_{b}^{*}$ are thus numerically confirmed, in particular, the decrease in the quantum barrier height along $E_{b}^{\ddagger} / E_{b}$ in Figs. 1(e) and 1(f) when $E_{\lambda}=0$ is due to the dominance of the second term in Eq. (22). These observations are behind the main results that will be shown later in Fig. 3.

Figure 2 compares the analytical and numerical solutions. It is seen that the deviation is large around $E_{\lambda} / E_{b} \simeq 8$ naturally because the premises of the approximations do not apply there. We also see finite deviations outside that region, which come from the neglected terms of higher and lower orders in $\delta$. In the limit $E_{\lambda} \rightarrow \infty$, the analytical and numerical solutions exactly agree, as we find $\delta \rightarrow 0$ [see Eqs. (A5)-(A7) in the Appendix], and both converge to the classical value $E_{b}$ as in Eq. (20). On the other hand, in the limit $E_{\lambda} \rightarrow 0$, a finite deviation remains because $\delta$ does not go to infinity as the system is bound in a potential. It is actually straightforward to evaluate this deviation to the second order in $\hbar$, which was found to largely improve the agreement for a model without the $Q$ mode. $^{30}$

\section{KINETIC ISOTOPE EFFECT}

\section{A. Analytical solution}

We now analyze the KIE, $k_{\mathrm{SQGH}}(\mathrm{D}) / k_{\mathrm{SQGH}}(\mathrm{H})$ $\simeq \exp \left\{-\beta\left(E_{b}^{*}(\mathrm{D})-E_{b}^{*}(\mathrm{H})\right)\right\}$, which is influenced by the couplings to the external degrees of freedom. In the weak coupling case, Eq. (19) gives

$$
E_{b, \text { weak }}^{*}(\mathrm{D})-E_{b, \text { weak }}^{*}(\mathrm{H})=\frac{1}{2} \hbar\left(\omega_{\mathrm{H}}-\omega_{\mathrm{D}}\right) \sqrt{\frac{E_{b}}{E_{b}^{*}}+\frac{1}{16} \frac{E_{\lambda}}{E_{b}}},
$$

where $\omega_{\mathrm{H}, \mathrm{D}}=\sqrt{2 a / m_{\mathrm{H}, \mathrm{D}}}$. Since $\omega_{\mathrm{H}}>\omega_{\mathrm{D}}$, we find that $E_{b \text {,weak }}^{*}(\mathrm{D})-E_{b \text {,weak }}^{*}(\mathrm{H})$ increases monotonically along the reorganization energy $E_{\lambda}$. On the other hand, in the strong coupling case, Eq. (20) gives

$$
\begin{aligned}
E_{b, \mathrm{str}}^{*}(\mathrm{D})-E_{b, \mathrm{str}}^{*}(\mathrm{H})= & \frac{1}{2} \hbar\left(\omega_{\mathrm{H}}-\omega_{\mathrm{D}}\right)\left[\sqrt{\frac{E_{b}}{E_{b}^{*}}+\frac{1}{16} \frac{E_{\lambda}}{E_{b}}}\right. \\
& -\sqrt{\left.\frac{1}{2}\left(\frac{1}{8} \frac{E_{\lambda}}{E_{b}}-1\right)\right]} .
\end{aligned}
$$

When $E_{\lambda}$ is sufficiently large, we may approximate the last factor as

$$
\sqrt{\frac{E_{b}}{E_{b}^{\ddagger}}+\frac{1}{16} \frac{E_{\lambda}}{E_{b}}}-\sqrt{\frac{1}{2}\left(\frac{1}{8} \frac{E_{\lambda}}{E_{b}}-1\right)} \simeq \sqrt{\frac{E_{b}}{E_{\lambda}}}\left(1+\frac{2 E_{b}}{E_{b}^{\ddagger}}\right),
$$

indicating that $E_{b \text {,str }}^{*}(\mathrm{D})-E_{b, \text { str }}^{*}(\mathrm{H})$ is a monotonically decreasing function of $E_{\lambda}$. It follows that $E_{b}^{*}(\mathrm{D})-E_{b}^{*}(\mathrm{H})$ should exhibit a maximum along $E_{\lambda}$. We note that this was found from the two limits, $E_{\lambda} \rightarrow 0$ and $E_{\lambda} \rightarrow \infty$, where the analytical expressions of $E_{b \text {,weak }}^{*}$ and $E_{b, \text { str }}^{*}$ are the most accurate. Obviously, the picture carries over for the $\mathrm{H} / \mathrm{T}$ and $\mathrm{D} / \mathrm{T}$ isotope effects.

\section{B. Numerical analysis}

The approximate analytical arguments in the previous section is confirmed by the numerical solutions displayed in Fig. 3. It is seen that $E_{b}^{*}(\mathrm{D})-E_{b}^{*}(\mathrm{H})$ has a maximum for the range of $E_{b}^{\ddagger}$ values examined. We also compare in Fig. 4 the analytical and numerical solutions. The quantitative discrepancy is due to the neglected terms of $\left(\hbar \omega / E_{b}\right)^{2}$ and the higher orders in $\hbar$. Nonetheless, the analytical solutions promptly describe the qualitative behavior in the weak and strong coupling regions. As noted in Fig. 2, the analytical and numerical solutions exactly agree in the $E_{\lambda} \rightarrow \infty$ limit, while a finite (and correctable) deviation remains in $E_{\lambda} \rightarrow 0$, and the KIE vanishes in the former where the barrier height reduces to the classical value.

\section{CONCLUDING REMARKS}

In this work, a theory for the adiabatic $\mathrm{H}$ transfer rate has been developed by combining the SQTDH theory and the MTST, taking into account the zero-point effects and the nonlocal spreading of the wavefunction in the adiabatic regime coupled to the external degrees of freedom. A set of compact analytical formulas has been derived in the limits of 

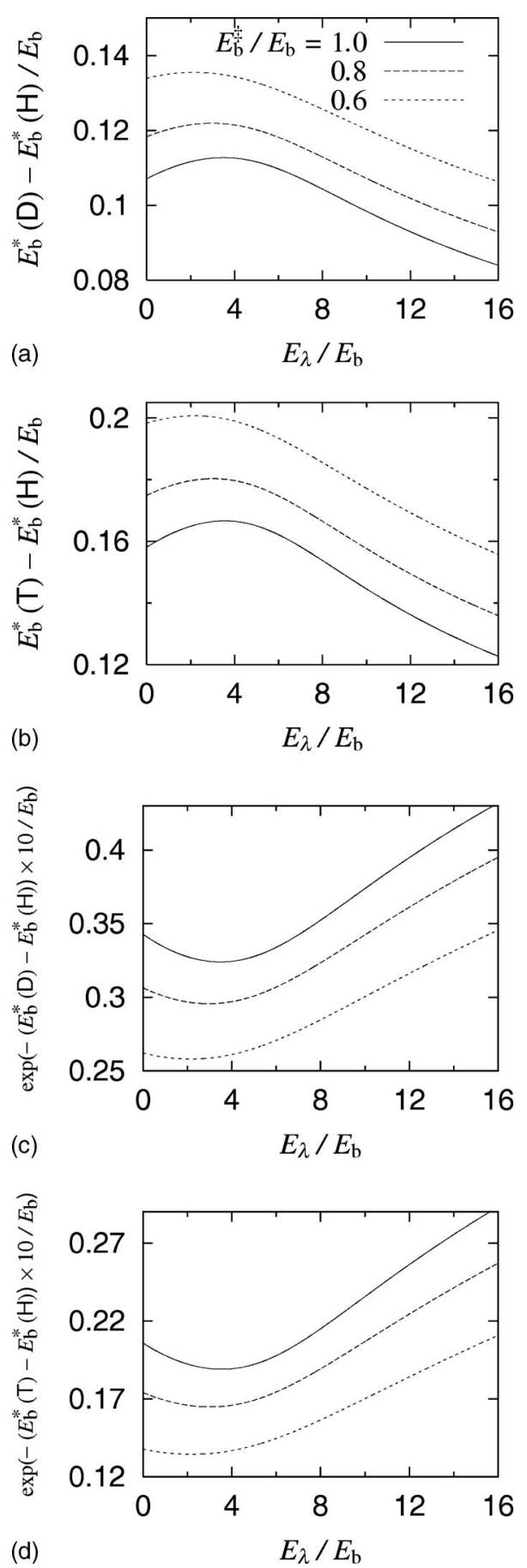

FIG. 3. Numerical solutions for the isotope effect $E_{b}^{*}(\mathrm{D}, \mathrm{T})-E_{b}^{*}(\mathrm{H})[(\mathrm{a})$ and (b) $]$ and $k_{\mathrm{SQGH}}(\mathrm{D}, \mathrm{T}) / k_{\mathrm{SQGH}}(\mathrm{H})$ at the temperature $k_{B} T=E_{b} / 10[(\mathrm{c})$ and (d)].

weak and strong couplings to the environment. The analysis has suggested that the KIE on the adiabatic $\mathrm{H}$ transfer rate may exhibit a maximum as a function of the coupling strength to the external degrees of freedom. Although we have not yet seen experimental evidence or signature, this might be observed with controlled external pressure ${ }^{67}$ and viscosity ${ }^{68,69}$ A more detailed analysis for its origin and the conditions in which to enhance or suppress the peak would be needed to offer further clues for experimental examinations.

It is also desired to extend the approach toward more realistic and complex systems than the model of Eq. (7),

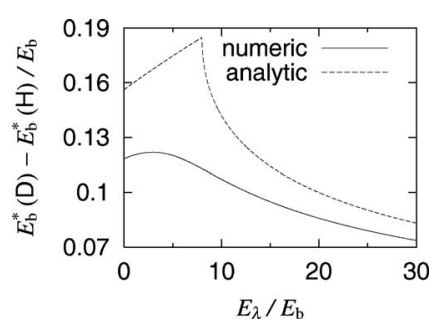

FIG. 4. Comparison between the numerical and analytical solutions of $E_{b}^{*}(\mathrm{D})-E_{b}^{*}(\mathrm{H})$ for $E_{b}^{\ddagger} / E_{b}=0.8$.

which would be possible in various ways. One of the most straightforward would be to generalize from the quartic double well to more generic forms of the potential. In this regard, encouraging results have been obtained on the structural correlations and the geometric isotope effects of the hydrogen bond structure. ${ }^{46}$ Another direction in which to proceed would be to combine the SQTDH theory with the reaction path/surface Hamiltonian approaches, ${ }^{11,12,18,19}$ because their application to the adiabatic regime seems rather scarce. Moreover, in cases where the choice of the reaction coordinate is not as obvious, e.g., in the collective multiple proton transfers in protic liquids, it will become necessary to properly account for the correlations among different degrees of freedom. While the thawed Gaussian wavepacket approach ${ }^{70}$ which is closely related to the present model, will appropriately handle this aspect, analogous treatment in the present framework will require additional considerations and approximations in order to maintain the extended Hamiltonian form. Works along this issue with applications are currently under way and will be reported elsewhere. We envisage that the present model study would serve as a basic reference for these extensive applications.

\section{ACKNOWLEDGMENTS}

K.A. acknowledges support from the Royal Society (G503/22211) and KAKENHI (19029026, in Priority Area "Molecular Theory for Real Systems").

\section{APPENDIX: NORMAL MODE ANALYSIS OF MTST}

We first minimize Eq. (9) along $X,\left\{X_{i}\right\}, \Delta$, and $\left\{\Delta_{i}\right\}$, which straightforwardly gives

$$
\begin{aligned}
V_{\mathrm{ext}}(x ; \delta)= & -\frac{a}{2} x^{2}+\frac{b}{4} x^{4}+\frac{1}{2}\left(-a+m \zeta(0)+3 B x^{2}\right) \delta^{2} \\
& +\frac{3 B}{4} \delta^{4}+\frac{\hbar^{2}}{8 m \delta^{2}}+E_{0},
\end{aligned}
$$

where we defined $B=b+4 D^{2} / 3 M \Omega^{2}$. $E_{0}$ is a constant consisting of the zero-point energies of the eliminated modes and other constants that are rather irrelevant for the current formulation, and hence will be omitted. The smaller number of arguments in $V_{\text {ext }}(x ; \delta)$ implies that the full $V_{\text {ext }}\left(x, X, X_{i} ; \delta, \Delta, \Delta_{i}\right)$ has been minimized along the omitted arguments, a convention which will be followed hereafter.

Reactant well bottom. The minimum of $V_{\text {ext }}(x ; \delta)$ along $x$ is found to be 


$$
V_{\mathrm{ext}}(\delta)=-\frac{3 B B^{\prime}}{2 b} \delta^{4}+\frac{1}{2} \alpha \delta^{2}-\frac{a^{2}}{4 b}+\frac{\hbar^{2}}{8 m} \frac{1}{\delta^{2}},
$$

in which we defined $B^{\prime}=b+2 D^{2} / M \Omega^{2}$ and $\alpha=2 a B^{\prime} / b$ $+m \zeta(0)$. Here, $\left(a-3 B \delta^{2}\right) / b>0$ is necessary for the minimum to exist, which we assume to be satisfied. Because the value of $\delta$ that gives the minimum of $V_{\text {ext }}$ is regarded to be small, ${ }^{30}$ we may approximate as

$$
V_{\mathrm{ext}}(\delta) \simeq \frac{1}{2} \alpha \delta^{2}-\frac{a^{2}}{4 b}+\frac{\hbar^{2}}{8 m} \frac{1}{\delta^{2}} .
$$

Therefore, the minimum is found at $\delta^{2}=\hbar / 2 \sqrt{m \alpha}$ to be

$$
V_{\mathrm{ext}} \simeq-\frac{a^{2}}{4 b}+\frac{\hbar}{2} \sqrt{\frac{\alpha}{m}} .
$$

Saddle point: Weak bath coupling case. Since the saddle point is located at $x=0$ due to the symmetry of the present model, we shall minimize along $\delta$,

$$
V_{\text {ext }}(x=0 ; \delta)=\frac{3 B}{4} \delta^{4}-\frac{a-m \zeta(0)}{2} \delta^{2}+\frac{\hbar^{2}}{8 m \delta^{2}} .
$$

The solution depends on the strength of the coupling to the bath via the sign of $a-m \zeta(0)$.
When the coupling to the bath is weak, the value of $\delta$ yielding the saddle point becomes large, so we may neglect the last term $\hbar^{2} / 8 m \delta^{2}$ in Eq. (A5). Therefore, with the weak coupling condition $m \zeta(0) \ll a$, we find the saddle point potential at $\delta^{2}=(a-m \zeta(0)) / 3 B$,

$$
V_{\mathrm{ext}}(x=0) \simeq-\frac{(a-m \zeta(0))^{2}}{12 B} .
$$

Saddle point: Strong bath coupling case. When the coupling to the bath is strong $[m \zeta(0) \gg a]$, the value of $\delta$ giving the saddle point becomes small and we may neglect the first term $(3 B / 4) \delta^{4}$ in Eq. (A5). The saddle point is then found to be

$$
V_{\mathrm{ext}}(x=0) \simeq \frac{\hbar}{2} \sqrt{\frac{m \zeta(0)-a}{m}}
$$

at $\delta^{2}=\hbar / 2 \sqrt{m \zeta(0)-a}$. In contrast to the weak coupling case, both $\delta^{2}$ and the barrier lowering are of order $\hbar^{1}$, and hence vanish in the classical limit $\hbar \rightarrow 0$.

Hessian matrix at reactant well. As noted in Sec. III B, we derive the Hessian matrices from $V_{\text {ext }}\left(x, X, X_{i}\right)$. After converting to the mass-weighted coordinates, $x^{\prime}=\sqrt{m} x$, $X^{\prime}=\sqrt{M} X$, and $X_{i}^{\prime}=\sqrt{M_{i}} X_{i}$, we find the Hessian matrix at the well bottom,

$$
\begin{aligned}
K_{\text {well }} \equiv & {\left[\begin{array}{ccccc}
\partial^{2} V_{\text {ext }} / \partial x^{\prime 2} & \partial^{2} V_{\text {ext }} / \partial x^{\prime} \partial X^{\prime} & \partial^{2} V_{\text {ext }} / \partial x^{\prime} \partial X_{1}^{\prime} & \ldots & \partial^{2} V_{\text {ext }} / \partial x^{\prime} \partial X_{N}^{\prime} \\
\partial^{2} V_{\text {ext }} / \partial X^{\prime} \partial x^{\prime} & \partial^{2} V_{\text {ext }} / \partial X^{\prime 2} & \partial^{2} V_{\text {ext }} / \partial X^{\prime} \partial X_{1}^{\prime} & \ldots & \partial^{2} V_{\text {ext }} / \partial X^{\prime} \partial X_{N}^{\prime} \\
\partial^{2} V_{\text {ext }} / \partial X_{1}^{\prime} \partial x^{\prime} & \partial^{2} V_{\text {ext }} / \partial X_{1}^{\prime} \partial X^{\prime} & \partial^{2} V_{\text {ext }} / \partial X_{1}^{\prime 2} & \ldots & \ldots \\
\ldots & \ldots & \ldots & \ldots & \ldots \\
\partial^{2} V_{\text {ext }} / \partial X_{N}^{\prime} \partial x^{\prime} & \partial^{2} V_{\text {ext }} / \partial X_{N}^{\prime} \partial X^{\prime} & \ldots & \ldots & \partial^{2} V_{\text {ext }} / \partial X_{N}^{\prime 2}
\end{array}\right] } \\
= & {\left[\begin{array}{ccccc}
\partial^{2} V_{\text {ext }} / \partial x^{\prime 2} & -2 D x_{\mathrm{well}}^{\prime} / \sqrt{m^{2} M} & -C_{1} / \sqrt{m M_{1}} & \ldots & -C_{N} / \sqrt{m M_{N}} \\
-2 D x_{\mathrm{well}}^{\prime} / \sqrt{m^{2} M} & \Omega^{2} & 0 & \ldots & 0 \\
-C_{1} / \sqrt{m M_{1}} & 0 & \Omega_{1}^{2} & \ldots & 0 \\
\ldots & \ldots & \ldots & \ldots & \ldots \\
-C_{N} / \sqrt{m M_{N}} & 0 & 0 & \ldots & \Omega_{N}^{2},
\end{array}\right], }
\end{aligned}
$$

in which

$$
\frac{\partial^{2} V_{\mathrm{ext}}}{\partial x^{\prime 2}}=\frac{1}{m}\left(2 a-\frac{3 B \hbar}{\sqrt{m \alpha}}+\frac{4 D^{2} x_{\mathrm{well}}^{\prime 2}}{m M \Omega^{2}}+\sum_{i=1}^{N} \frac{C_{i}^{2}}{M_{i} \Omega_{i}^{2}}\right)
$$

and

$$
x_{\mathrm{well}}^{\prime 2}=\frac{m}{2 b}\left(2 a-\frac{3 B \hbar}{\sqrt{m \alpha}}\right) .
$$

Therefore, we find

$$
\operatorname{det} K_{\mathrm{well}}=\frac{1}{m}\left(2 a-\frac{3 B \hbar}{\sqrt{m \alpha}}\right) \Omega^{2} \prod_{i=1}^{N} \Omega_{i}^{2} .
$$

Hessian matrix at saddle point. As the saddle point is located at $x=0$, the symmetrically coupled mode $X^{\prime}$ is decoupled from the other degrees of freedom, so it is convenient to rearrange the rows and columns as 


$$
\begin{aligned}
K_{\text {sad,weak }} \equiv & {\left[\begin{array}{ccccc}
\partial^{2} V_{\text {ext }} / \partial X^{\prime 2} & \partial^{2} V_{\text {ext }} / \partial X^{\prime} \partial x^{\prime} & \partial^{2} V_{\text {ext }} / \partial X^{\prime} \partial X_{1}^{\prime} & \ldots & \partial^{2} V_{\text {ext }} / \partial X^{\prime} \partial X_{N}^{\prime} \\
\partial^{2} V_{\text {ext }} / \partial x^{\prime} \partial X^{\prime} & \partial^{2} V_{\text {ext }} / \partial x^{\prime 2} & \partial^{2} V_{\text {ext }} / \partial x^{\prime} \partial X_{1}^{\prime} & \ldots & \partial^{2} V_{\text {ext }} / \partial x^{\prime} \partial X_{N}^{\prime} \\
\partial^{2} V_{\text {ext }} / \partial X_{1}^{\prime} \partial X^{\prime} & \partial^{2} V_{\text {ext }} / \partial X_{1}^{\prime} \partial x^{\prime} & \partial^{2} V_{\text {ext }} / \partial X_{1}^{\prime 2} & \ldots & \ldots \\
\ldots & \ldots & \ldots & \ldots & \ldots \\
\partial^{2} V_{\text {ext }} / \partial X_{N}^{\prime} \partial X^{\prime} & \partial^{2} V_{\text {ext }} / \partial X_{N}^{\prime} \partial x^{\prime} & \ldots & \ldots & \partial^{2} V_{\text {ext }} / \partial X_{N}^{\prime 2}
\end{array}\right] } \\
& =\left[\begin{array}{cccccc}
\Omega^{2}-\frac{2 D^{2}}{3 M B^{\prime}} & 0 & 0 & 0 & \ldots & 0 \\
0 & -\zeta(0)+\sum_{i=1}^{N} \frac{C_{i}^{2}}{M_{i} \Omega_{i}^{2}} & -C_{1} / \sqrt{m M_{1}} & -C_{2} / \sqrt{m M_{2}} & \ldots & -C_{N} / \sqrt{m M_{N}} \\
0 & -C_{1} / \sqrt{m M_{1}} & \Omega_{1}^{2} & 0 & \ldots & 0 \\
0 & -C_{2} / \sqrt{m M_{2}} & 0 & \Omega_{2}^{2} & \ldots & 0 \\
\ldots & \ldots & \ldots & \ldots & \ldots & \ldots \\
0 & -C_{N} / \sqrt{m M_{N}} & 0 & 0 & \ldots & \Omega_{N}^{2}
\end{array}\right] .
\end{aligned}
$$

Similarly, in the strong coupling case, we find

$$
K_{\text {sad,str }}=\left[\begin{array}{cccccc}
\Omega^{2} & 0 & 0 & 0 & \cdots & 0 \\
0 & \partial^{2} V_{\text {ext }} / \partial x^{\prime 2} & -C_{1} / \sqrt{m M_{1}} & -C_{2} / \sqrt{m M_{2}} & \cdots & -C_{N} / \sqrt{m M_{N}} \\
0 & -C_{1} / \sqrt{m M_{1}} & \Omega_{1}^{2} & 0 & \ldots & 0 \\
0 & -C_{2} / \sqrt{m M_{2}} & 0 & \Omega_{2}^{2} & \ldots & 0 \\
\cdots & \cdots & \ldots & \cdots & \ldots & \ldots \\
0 & -C_{N} / \sqrt{m M_{N}} & 0 & 0 & \cdots & \Omega_{N}^{2}
\end{array}\right],
$$

where

$$
\frac{\partial^{2} V_{\mathrm{ext}}}{\partial x^{\prime 2}}=-\frac{1}{m}\left(a-\frac{3 B \hbar}{2 \sqrt{m(m \zeta(0)-a)}}+\sum_{i=1}^{N} \frac{C_{i}^{2}}{M_{i} \Omega_{i}^{2}}\right) .
$$

Due to the decoupling of the $X^{\prime}$, the treatment is in part similar to the classical Grote-Hynes case. We find the following for the weak and strong coupling cases:

$$
\operatorname{det} K_{\text {sad,weak }}=-\frac{1}{m}\left(\Omega^{2}-\frac{2 D^{2}}{3 M B^{\prime}}\right) m \zeta(0) \prod_{i=1}^{N} \Omega_{i}^{2}
$$

and

$$
\operatorname{det} K_{\mathrm{sad}, \mathrm{str}}=-\frac{1}{m}\left(a-\frac{3 B \hbar}{2 \sqrt{m(m \zeta(0)-a)}}\right) \Omega^{2} \prod_{i=1}^{N} \Omega_{i}^{2} .
$$

Reaction rate. Given the eigenvalues of the Hessian matrices in terms of the mass-weighted coordinates at the reactant well bottom and the saddle point, $\left\{\left(\lambda_{\text {well }}^{(0)}\right)^{2}, \ldots\right\}$ and $\left\{-\mu^{2},\left(\lambda_{\text {sad }}^{(1)}\right)^{2}, \ldots\right\}$, the reaction rate is expressed as

$$
\begin{aligned}
k_{\mathrm{SQGH}} & =\frac{1}{2 \pi} \frac{\prod_{i=0} \lambda_{\mathrm{well}}^{(i)}}{\prod_{i=1} \lambda_{\mathrm{sad}}^{(i)}} \exp \left(-\beta E_{b}^{*}\right) \\
& =\frac{\mu}{2 \pi} \sqrt{\frac{\operatorname{det} K_{\mathrm{well}}}{-\operatorname{det} K_{\mathrm{sad}}}} \exp \left(-\beta E_{b}^{*}\right),
\end{aligned}
$$

in which the reactive frequency $\mu$ is determined from Eqs. (13) and (17). The classical limit of this framework ${ }^{31,32}$ yields the result equivalent to the Grote-Hynes theory.

${ }^{1}$ A. M. Kuznetsov and J. Ulstrup, Chem. Phys. 188, 131 (1994).

${ }^{2}$ A. Staib, D. Borgis, and J. T. Hynes, J. Chem. Phys. 102, 2487 (1995).

${ }^{3}$ K. Ando and J. T. Hynes, Adv. Chem. Phys. 110, 381 (1999).

${ }^{4}$ A. L. Sobolewski and W. Domcke, Chem. Phys. 184, 115 (1994).

${ }^{5}$ P. Kiefer and J. T. Hynes, J. Phys. Chem. A 106, 1834 (2002).

${ }^{6}$ P. Kiefer and J. T. Hynes, J. Phys. Chem. A 106, 1850 (2002).

${ }^{7}$ P. Kiefer and J. T. Hynes, J. Phys. Chem. A 107, 9022 (2003).

${ }^{8}$ W. W. Cleland and M. Kreevoy, Science 264, 1887 (1994).

${ }^{9}$ P. A. Frey, S. A. Whitt, and J. B. Tobin, Science 264, 1927 (1994).

${ }^{10}$ N. C. Schutz and A. Warshel, Proteins 55, 711 (2004).

${ }^{11}$ T. Carrington and W. H. Miller, J. Chem. Phys. 84, 4364 (1986).

${ }^{12}$ N. Shida, P. F. Barbara, and J. Almlof, J. Chem. Phys. 91, 4061 (1989).

${ }^{13}$ S. Hammes-Schiffer and J. C. Tully, J. Chem. Phys. 101, 4657 (1994).

${ }^{14}$ R. I. Cukier and D. Nocera, Annu. Rev. Phys. Chem. 49, 337 (1998).

${ }^{15}$ R. L. Redington, J. Chem. Phys. 113, 2319 (2000).

${ }^{16}$ C. S. Tautermann, A. F. Voegele, T. Loerting, and K. R. Liedl, J. Chem. Phys. 117, 1962 (2002).

${ }^{17}$ A. Ishizaki and Y. Tanimura, J. Chem. Phys. 123, 014503 (2005).

${ }^{18}$ K. Giese and O. Kühn, J. Chem. Phys. 123, 054315 (2005).

${ }^{19}$ D. P. Tew, N. C. Handy, and S. Carter, J. Chem. Phys. 125, 084313 (2006). 
${ }^{20}$ H. Sumi and J. Ulstrup, Biochim. Biophys. Acta 955, 26 (1988).

${ }^{21}$ D. Borgis, S. Lee, and J. T. Hynes, Chem. Phys. Lett. 162, 19 (1989).

${ }^{22}$ D. Borgis and J. T. Hynes, Chem. Phys. 170, 315 (1993).

${ }^{23}$ D. Borgis and J. T. Hynes, J. Phys. Chem. 100, 1118 (1996).

${ }^{24}$ S. Hammes-Schiffer, Acc. Chem. Res. 34, 273 (2001).

${ }^{25}$ M. P. Meyer and J. P. Klinman, Chem. Phys. 319, 283 (2005).

${ }^{26}$ V. G. Levich, Physical Chemistry-An Advanced Treatise (Academic, New York, 1970).

${ }^{27}$ J. Ulstrup, Charge Transfer Processes in Condensed Media (Springer, Berlin, 1979).

${ }^{28}$ R. A. Marcus and N. Sutin, Biochim. Biophys. Acta 811, 265 (1985).

${ }^{29}$ K. Ando, J. Chem. Phys. 121, 7136 (2004).

${ }^{30}$ K. Ando, Chem. Phys. Lett. 376, 532 (2003).

${ }^{31}$ E. Pollak, J. Chem. Phys. 85, 865 (1986).

${ }^{32}$ P. Hänggi, P. Talkner, and M. Borkovec, Rev. Mod. Phys. 62, 251 (1990).

${ }^{33}$ R. F. Grote and J. T. Hynes, J. Chem. Phys. 73, 2715 (1980).

${ }^{34}$ J. P. Bergsma, B. J. Gertner, K. R. Wilson, and J. T. Hynes, J. Chem. Phys. 86, 1356 (1987).

${ }^{35}$ R. P. Bell, The Tunnel Effect in Chemistry (Chapman \& Hall, London, 1980).

${ }^{36}$ L. Melander and W. H. Saunders, Reaction Rates of Isotopic Molecules (Wiley, New York, 1980).

${ }^{37}$ M. J. Knapp and J. P. Klinman, Eur. J. Biochem. 269, 3113 (2002).

${ }^{38}$ A. Kohen, Prog. React. Kinet. Mech. 28, 119 (2003).

${ }^{39}$ H. H. Limbach, J. M. Lopez, and A. Kohen, Philos. Trans. R. Soc. London, Ser. B 361, 1399 (2006).

${ }^{40} \mathrm{P}$. Kramer and M. Saraceno, Geometry of the Time-Dependent Variational Principle in Quantum Mechanics (Springer, Berlin, 1981).

${ }^{41}$ J. Broeckhove, L. Lathouwers, E. Kesteloot, and P. van Leuven, Chem. Phys. Lett. 149, 547 (1988).

${ }^{42}$ F. Arickx, J. Broeckhove, E. Kesteloot, L. Lathouwers, and P. van Leuven, Chem. Phys. Lett. 128, 310 (1986).

${ }^{43}$ Y. Tsue, Prog. Theor. Phys. 88, 911 (1992).

${ }^{44}$ A. K. Pattanayak and W. C. Schieve, Phys. Rev. E 50, 3601 (1994).

${ }^{45}$ K. Ando, Phys. Rev. B 72, 172104 (2005).

${ }^{46}$ K. Ando, J. Chem. Phys. 125, 014104 (2006).

${ }^{47}$ N. Makri and W. H. Miller, J. Chem. Phys. 87, 5781 (1987).

${ }^{48}$ O. V. Prezhdo, J. Chem. Phys. 117, 2995 (2002).
${ }^{49}$ E. Heatwole and O. V. Prezhdo, J. Chem. Phys. 126, 204108 (2007).

${ }^{50}$ Y. Shigeta, H. Miyachi, and K. Hirao, J. Chem. Phys. 125, 244102 (2006).

${ }^{51}$ P. G. Wolynes, Phys. Rev. Lett. 47, 968 (1981).

${ }^{52}$ M. Topaler and N. Makri, J. Chem. Phys. 101, 7500 (1994).

${ }^{53}$ I. R. Craig, M. Thoss, and H. Wang, J. Chem. Phys. 127, 144503 (2007).

${ }^{54}$ E. Bosch, M. Moreno, J. M. Lluch, and J. Bertrán, J. Chem. Phys. 93, 5685 (1990).

${ }^{55}$ D. Borgis and J. T. Hynes, J. Chem. Phys. 94, 3619 (1991).

${ }^{56}$ S. Takada and H. Nakamura, J. Chem. Phys. 100, 98 (1994).

${ }^{57}$ D. Antoniou and S. D. Schwartz, J. Chem. Phys. 108, 3620 (1998).

${ }^{58}$ S. Caratzoulas and S. D. Schwartz, J. Chem. Phys. 114, 2910 (2001).

${ }^{59}$ Q. Cui and M. Karplus, J. Phys. Chem. B 106, 7927 (2002).

${ }^{60}$ K. Ando and J. T. Hynes, J. Phys. Chem. B 101, 10464 (1997).

${ }^{61}$ K. Ando and J. T. Hynes, J. Phys. Chem. A 103, 10398 (1999).

${ }^{62}$ A. K. Pattanayak and W. C. Schieve, Phys. Rev. E 56, 278 (1997).

${ }^{63}$ Accounts of higher order moments or cumulants (Ref. 50) will not alter the qualitative picture either because the wavepacket broadening at the saddle point is essentially determined by the balance between the negative potential curvature at the saddle point and the positive ones in the wall regions [represented by the $b q^{4} / 4$ term in $V_{0}(q)$ in the present model].

${ }^{64}$ M. J. Gillan, J. Phys. C 20, 3621 (1987).

${ }^{65}$ D. H. Li and G. A. Voth, J. Phys. Chem. 95, 10425 (1991).

${ }^{66}$ In practice, macroscopic classical systems would be normally affected by external degrees of freedom such that we shall consider $\beta \hbar \omega \rightarrow 0$ for the classical limit, where $\omega$ characterizes the time scale of the system $(q)$ motion [as that in Eq. (18) in the current model]. In this case, the strong coupling regime would appropriately apply and thus the classical barrier height is recovered.

${ }^{67}$ H. Park, G. Kidman, and D. B. Northrop, Arch. Biochem. Biophys. 433, 335 (2005).

${ }^{68}$ A. Mordzinski, J. Lipkowski, and G. Orzanowska, Chem. Phys. 140, 167 (1990).

${ }^{69}$ C. J. Jalink, A. H. Huizer, and C. A. G. O. Varma, J. Chem. Soc., Faraday Trans. 86, 3717 (1990).

${ }^{70}$ E. J. Heller, J. Chem. Soc., Faraday Trans. 62, 1544 (1975). 